\title{
Parenthetical constructions in Russian spoken discourse: Basic types and prosodic features
}

\author{
Nikolay A. Korotaev \\ Russian State University for the Humanities \\ (RSUH) \\ n korotaev@hotmail.com
}

\begin{abstract}
The paper discusses the notion of parentheticals in Russian spoken discourse. Using data from two prosodically annotated corpora - "Stories about presents and skiing" and "Russian Pear Chats \& Stories" - I advocate for a discourse-oriented approach to parenthetical constructions. I define a parenthetical construction as consisting of three elements: the left context, the parenthetical unit, and the right context. Each element constitutes a separate discourse unit and is thus prosodically autonomous. I rely on the notion of projection [Auer 2005] to account for the discourse relationships between these three components. When the speaker pronounces the left context, she projects a continuation that is to be realized in the right context, while the parenthetical unit provides a digressive discourse step.

Typically (around 50\% in my data), parentheticals are anchored to their left contexts and are pronounced with a falling or level pitch accent. Noted deviations from this prototype include free parentheticals, parenthetical uses of $v o t$, and parentheticals pronounced with a rising pitch accent. Furthermore, I explore two prosodic features frequently associated with parentheticals, namely, increased articulation rate and pitch range narrowing. I show that, while both these tendencies are statistically significant, the latter has a larger effect size than the former.

Keywords: spoken discourse, Russian language, discourse structure, discourse prosody, parentheticals, intonation, speech rate, pitch range
\end{abstract}

DOI: $10.28995 / 2075-7182-2021-20-413-424$

\section{Конструкции с дискурсивными вставками в устной русской речи: базовые типы и просодические свойства}

\author{
Николай Коротаев \\ Российский государственный гуманитарный \\ университет (РГГУ) \\ n korotaevahotmail.com
}

\begin{abstract}
Ключевые слова: устная речь, русский язык, структура дискурса, дискурсивная просодия, парентеза, интонация, темп, тональный диапазон
\end{abstract}

\section{1 Вводные замечания}

Данная работа посвящена явлению дискурсивной вставки (парентезы) в неподготовленной устной речи. Под конструкцией со вставкой я, вслед за [Кибрик, Подлесская 2010], буду понимать последовательность из как минимум трех дискурсивных единиц: первая и последняя связаны между собой тем или иным содержательным отношением в рамках основной линии изложения, а между ними располагается фрагмент, выбивающийся из основной линии. С точки зрения динамического развертывания дискурса особенность таких конструкций заключается в том, что говорящий временно откладывает намеченное в первой единице продолжение, реализует побочное действие, а затем возвращается к изначальному плану. Так, в примере (1) говорящий, произнеся единицу $\mathrm{E} 010^{1}$, недвусмысленно указывает на планируемое продолжение: для этого

\footnotetext{
${ }^{1}$ Об оформлении примеров и используемых в них обозначениях см. раздел 2.
} 
используются как синтаксические (деепричастная форма), так и интонационные (восходящий тональный акцент) средства.

(1) Pic-RUS 05-m Ski-T

\begin{tabular}{|c|c|c|}
\hline 19.57 & E010 & /Выпив хорошенько, \\
\hline 20.83 & p-007 & $(0.18)$ \\
\hline 21.02 & E011 & (\Непонятно, \\
\hline 21.71 & $\mathrm{p}-008$ & $(0.05)$ \\
\hline 21.76 & E012 & почему (0.05) кстати с \утра он пьёт.) \\
\hline 23.65 & p-009 & $(0.78)$ \\
\hline 24.42 & E013 & Лон (0.42) решил покататься ещё на \лыжах, \\
\hline 26.98 & E014 & и /видимо нь = $\|$ выбрал не ту $-\downarrow$ горку. \\
\hline
\end{tabular}

Намеченное продолжение реализуется в строке Е013, которая образует с Е010 единый содержательный, синтаксический и интонационный комплекс. При этом между этими двумя единицами производится вставка: комментарий рассказчика не входит в основную линию изложения и - что еще более существенно - не может восприниматься как завершение ранее начатого высказывания. В транскрипте вставленные единицы заключены в скобки. На рис. 1 представлена тонограмма этого фрагмента: можно заметить, что единицы Е011-Е012 произносятся с ощутимо менее рельефными движениями частоты основного тона, чем единицы $\mathrm{E} 010$ и Е $013^{2}$.

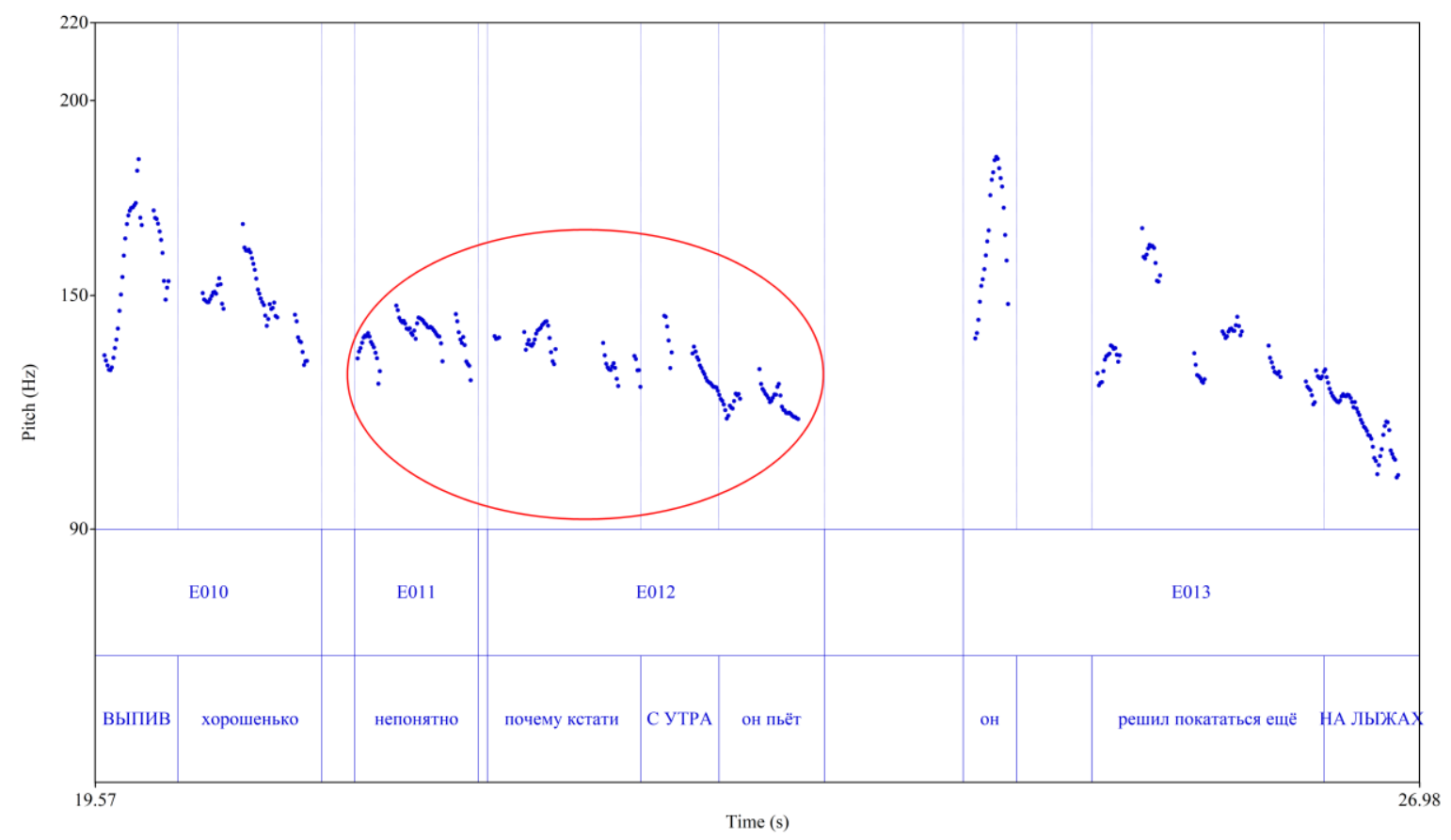

Рис. 1. Тонограмма примера (1). Заглавными буквами выделены словоформы, произносимые с главными акцентами; овалом - участок тонограммы, соответствующий вставке

Вставкам посвящена обширная литература, см., в частности, [Urmson 1952; McCawley 1982; Кобозева 1999; Гавриленко 2004; Dehé \& Kavalova (eds.) 2007; Mazeland 2007; Падучева 2010: 321-334; Богданова 2011; Dehé 2014]. Сколько-либо подробное обсуждение этих источников выходит за рамки настоящей работы, однако необходимо отметить, что единого понимания парентезы, по всей видимости, не существует. Вставка - это многофакторное явление, при описании которого, в зависимости от исследовательских задач, на первый план выходят соображения семантико-прагматического, синтаксического, просодического, дискурсивного и

\footnotetext{
${ }^{2}$ Акустический анализ примеров выполнялся в программе Praat [Boersma \& Weenink 2018].
} 
иного характера. Для настоящей работы центральным является вопрос о том, каким образом вставки реализуются в структуре неподготовленного устного русского текста. Непосредственные задачи исследования сводятся к следующему:

- сформулировать рабочее определение конструкции со вставкой и продемонстрировать, какие классы конструкций выделяются в привлеченном устном материале;

- реализовать предварительный анализ акустических характеристик вставленных элементов.

Дальнейший текст статьи имеет следующую структуру. В разделе 2 будет описан материал исследования и кратко представлены базовые принципы используемой дискурсивной нотации. В разделе 3 будут рассмотрены интегральные свойства конструкций со вставками, на которые я предлагаю опираться при определении. В разделе 4 я покажу, какие базовые классы конструкций со вставками выделяются в корпусе, и рассмотрю их дифференцирующие свойства. В разделе 5 будут представлены предварительные результаты количественного анализа темповых и тональных характеристик вставок. Раздел 6 содержит заключительные замечания.

\section{2 Материал}

Материалом исследования послужили записи двух корпусов звучащей речи, снабженных дискурсивно-просодической разметкой:

- «Истории о подарках и катании на лыжах» (http://spokencorpora.ru/showcorpus.py?dir=03pands/rus) - 35 минут звучания, около 4500 словоупотреблений;

- три записи корпуса «Рассказы и разговоры о грушах» (https://multidiscourse.ru/; [Кибрик 2018]) - 60 минут звучания, около 10000 словоупотреблений ${ }^{3}$

Все записи содержат речь носителей русского языка (от 18 до 30 лет): исключительно монологического (нарративного) характера в первом корпусе и смешанного диалого-монологического - во втором. На начало проведения исследования все записи имели доступную разметку. Перечислю базовые особенности текстового компонента разметки (= дискурсивной транскрипции), существенные для понимания приводимых в статье примеров; более подробное описание принципов аннотации см. в [Kibrik et al. 2020].

- Транскрипты поделены на нумерованные строки. Строки, номер которых содержит литеру Е, соответствуют элементарным дискурсивным единицам (ЭДЕ). ЭДЕ — это минимальные шаги в развитии устного дискурса, выделяемые на основании набора просодических критериев.

- В каждой ЭДЕ (за возможным исключением коротких оборванных единиц) содержится по крайней мере одна акцентированная, т.е. просодически выделенная, словоформа. Наличие акцентов отмечается в транскрипте при помощи слэшей и стрелок перед акцентированными словоформами; при этом направление слэшей указывает на характер движения частоты основного тона в ударном слоге, направление стрелок - на значимые внеударные движения. Например, словоформа /выпив в ЭДЕ Е010 примера (1) произносится с восходящим акцентом; а в словоформе - $\downarrow$ горку (ЭДЕ Е014) реализован ровно-нисходящий акцент, причем нисходящая часть расположена на заударном слоге.

- Подчеркиванием ударной гласной дополнительно отмечаются главные акценты в ЭДЕ. Направление движения тона в главном акценте является одним из центральных факторов, определяющих иллокутивно-фазовое значение ЭДЕ, которое, в свою очередь, кодируется при помощи пунктуационных знаков в конце строки. Выделяются две группы пунктуационные знаков: финальные (прежде всего, точка) и нефинальные (запятая и некоторые

\footnotetext{
${ }^{3}$ В заголовках примеров указывается кодовое имя записи. Коды, начинающиеся с Pic-RUS, соответствуют рассказам корпуса «Истории о подарках и катании на лыжах»; коды, начинающиеся с Pears, — записям корпуса «Рассказы и разговоры о грушах».
} 
другие). Это разграничение связано с противопоставлением завершенности vs. незавершенности, подробно рассматриваемым, в частности, в работах [Янко 2008; Кодзасов 2009].

\section{3 Интегральные свойства и рабочее определение}

Как уже было отмечено в разделе 1 , в данной работе за основу взято определение вставки из [Кибрик, Подлесская 2010]. Приведу фрагмент этого определения, также сформулированного на устном корпусном материале: «Вставка - это временное отклонение от маршрута изложения, выполнение некоторого побочного хода, и затем возврат к исходному маршруту. (...) ... последовательность из трех дискурсивных единиц $\mathrm{X}+\mathrm{Y}+\mathrm{Z}$, в которой $\mathrm{Y}$ - уточнение к $\mathrm{X}$, а основная линия повествования связывает $X$ и $Z$ ». Ниже я несколько подробнее рассмотрю компоненты этого определения и укажу на свойства, общие для всех вставочных конструкций, обнаруженных в корпусе.

\section{1 Трехчастная структура}

Для квалификации конструкции как содержащей вставку необходимо наличие трех компонентов: вставка не просто соотносится с некоторым контекстом, но и обязательно разрывает его. При таком понимании к вставкам не относятся коммуникативно подавленные элементы левой / правой периферии клаузы: начальные употребления вводных оборотов вида (как) кажется, постпозитивные расширения и проч. Подобные элементы в этой статье не рассматриваются. Далее для единиц, обозначенных выше как X, Y и Z, я буду использовать рабочие термины «левый контекст», «вставка» и «правый контекст». Так, в примере (1) в строке Е010 завершается левый контекст, строки Е011 и Е012 составляют вставку, а в строке Е013 начинается правый контекст ${ }^{4}$.

\section{2 Проекция на продолжение}

Для интерпретации того факта, что левый и правый контекст реализуют основную линию изложения, кажется удобным опираться на введенное П. Ауэром понятие проекции [Auer 2005]. Под проекцией понимается разделяемое участниками коммуникации представление о том, что одно речевое действие в известной степени влечет, или проецирует, другое. В конструкциях со вставками проекция возникает при произнесении левого контекста: реализуя вставку, говорящий подразумевает, что слушатель сохранит сформированные ранее ожидания продолжения до начала произнесения правого контекста.

Степень проекции может быть различной. В примере (1) был представлен случай сильной проекции, поскольку использование в левом контексте деепричастной клаузы с высокой степенью вероятности предсказывает дальнейшее появление главной клаузы. Соответственно, не получив ожидаемого структурного продолжения в строках Е011-012, слушатель интерпретирует эти ЭДЕ как вставку и «переносит» свои ожидания на правый контекст. Однако столь явные указания на характер продолжения имеются далеко не всегда. Так, в примере (2) строка R-vE164 содержит интонационный сигнал незавершенности (восходящее движение тона в главном акценте), но какими структурными единицами эта незавершенность должна «разрешаться», не специфицируется. Это можно назвать слабой проекцией.

(2) Pears23R

\begin{tabular}{lll}
\hline 792.12 & R-vE163 & для чего-то берёт последнюю/грушу, \\
\hline 793.72 & R-vE164 & снимает с себя /платок такой, \\
\hline 795.18 & pR-136 & $(0.11)$ \\
\hline 795.29 & R-vE165 & (\краснный вот, \\
\hline 795.96 & pR-137 & $(0.20)$ \\
\hline 796.16 & R-vE166 & который на -шею вешают,) \\
\hline
\end{tabular}

\footnotetext{
${ }^{4}$ В [Кибрик, Подлесская 2010] также упоминаются случаи «односторонней парентезы», при которых единицы, изначально порождаемые как вставки, берут на себя функцию правого контекста. Такие примеры встречаются и в нашем материале, но в настоящей работе не рассматриваются.
} 


\begin{tabular}{lll}
\hline 797.26 & $\mathrm{R}-\mathrm{vE} 167$ & протирает эту последнюю /грушу, \\
\hline 798.81 & $\mathrm{pR}-138$ & $(0.18)$ \\
\hline 798.98 & $\mathrm{R}-\mathrm{vE168}$ & и /-кладёт /-обратно в \корзину. \\
\hline
\end{tabular}

\section{3 Автономность вставки}

Вне зависимости от типа проекции вставленные единицы не могут интерпретироваться как полноценное продолжение структуры, проецируемой в левом контексте. Появление вставки сигнализирует о том, что ожидаемое продолжение откладывается. Вместе с тем, согласно приведенному определению, вставка все же представляет собой отдельный - пусть и побочный — дискурсивный шаг. Формально это выражается тем, что вставка имеет автономный интонационный контур и потому интерпретируется как ЭДЕ или группа ЭДЕ.

Отмечу, что аналогичный подход представлен в работах по анализу бытового диалога, в которых под парентезой понимаются отдельные репликообразующие единицы (turn-constructional units), также выделяемые на просодических основаниях [Mazeland 2007; Schegloff 2007: $237 \mathrm{ff}]$. Напротив, представление о парентезе как о безакцентном элементе коммуникативной структуры, синтаксически не связанном с объемлющим контекстом [Янко 2001: 81], с предлагаемым определением не согласуется. Как видно из примеров выше и ниже по тексту, вставки обладают интонационной автономностью и при этом могут быть как синтаксически независимы от левого и правого контекста (см. примеры (1), (5)), так и вступать с ним в те или иные синтаксические отношения $(2-4,6)$.

\section{4 Уровень локальной структуры}

Отдельным является вопрос об уровне иерархической структуры дискурса, на котором происходит вставка. В примерах (1) и (2) речь шла о вставке между ЭДЕ. Случай другого рода представлен в примере (3): здесь вставка интонационно автономной единицы C-vE113 происходит между составляющими простой клаузы.

(3) Pears 22C

\begin{tabular}{lll}
\hline 374.87 & C-vE111 & И он с этими Агрушами возвращается к своим /друзьям, \\
\hline 377.08 & C-vE112 & 个даёт им - \\
\hline 377.49 & C-vE113 & (\каждому, \\
\hline 377.89 & C-vE114 & - по /груше, \\
\hline 378.37 & C-vE115 & и /они идут мимо /фермера, \\
\hline 379.66 & C-vN036 & (ч 0.38) \\
\hline 380.04 & C-vE116 & /весело жуя эти -груши. \\
\hline
\end{tabular}

Дистрибутивное местоимение каждому формирует отдельную ЭДЕ, поскольку произносится с ощутимым акцентом, выбивающимся из единого интонационного контура клаузы даёт им по груше. Если бы каждому произносилось безударно или же вовсе не было произнесено, последовательность строк C-vE112-114 составляла бы одну ЭДЕ. Таким образом, можно сказать, что вставка здесь происходит не между, а внутри ЭДЕ. В [Кибрик, Подлесская 2010] для таких случаев используется термин «сплит»; в транскрипте левый и правый контекст маркируется при помощи длинного тире ${ }^{5}$. Особенность сплита состоит в том, что говорящий решает осуществить побочный дискурсивный шаг, не дожидаясь завершения текущего шага. Возможно, с этим связана достаточно частотная особенность конструкций со сплитом: в отличие от случаев (1)-(2), в которых вставки обладают «подавленным» коммуникативным статусом, вставка, попадающая в сплит, нередко, напротив, информационно выделена.

Как бы то ни было, и в примерах (1)-(2), и в примере (3) вставка происходит на уровне, не выходящем за рамки одного «устного предложения», т.е. реализуется в ситуации интонационно выраженной в левом контексте дискурсивной незавершенности. По всей видимости, вставки

\footnotetext{
${ }^{5}$ Аналогичное противопоставление между вставками, происходящими между репликообразующими единицами vs. внутри одной репликообразующей единицы, рассматривается на материале диалогических данных в [Mazeland 2007].
} 
возможны и на более высоком уровне дискурсивной структуры - между предложениями. Обособленный характер вставленного материала в таких случаях может быть, например, выражен лексически (кстати, между прочим и др.). Однако в общем случае, ввиду отсутствия интонационной проекции на продолжение после левого контекста, идентификация таких последовательностей как содержащих вставку значительно менее очевидна. В настоящей работе такие примеры не рассматриваются ${ }^{6}$.

Итак, при разметке корпуса использовалось следующее понимание конструкции с дискурсивной вставкой:

- конструкция состоит из трех компонентов: левого контекста, собственно вставки и правого контекста;

- левый контекст характеризуется дискурсивной незавершенностью и проецирует продолжение, реализуемое не во вставке, а в правом контексте;

- вставка представляет собой отдельную ЭДЕ или группу ЭДЕ.

\section{4 Типы конструкций в корпусе: дифференцирующие свойства}

Всего в исследованном подкорпусе обнаружено 236 конструкций с дискурсивными вставками: 174 случая в «Рассказах и разговорах о грушах» и 62 - в «Историях о подарках и катании на лыжах». Выше, при обсуждении интегральных свойств вставочных конструкций, были также упомянуты некоторые параметры варьирования: наличие и характер синтаксического отношения между вставкой и контекстом, объем вставки, тип проекции и др. Ниже будут рассмотрены еще две группы свойств, связанных (а) с характером содержательного отношения вставки к контексту и (б) с типом содержательной и интонационной обособленности вставки. Опора на эти параметры позволяет определить базовые типы конструкций с дискурсивными вставками в проанализированном материале.

\section{1 Ядерный тип}

Ядро выявленных случаев (к ним, в частности, относятся приведенные выше примеры (1) - (3)) составляют конструкции, характеризуемые следующим набором прототипических свойств.

(i) Вставка содержательно соотносится с конкретным элементом синтаксической структуры объемлющего контекста: с целой клаузой (пример (1)), с глагольной группой (3), с именной вершиной (2) и др. В [Kavalova 2007] для подобных случаев предлагается термин anchored parentheticals. Также существенно, что в прототипической ситуации «якорь», к которому содержательно прикрепляется вставка, располагается в левом, а не в правом контексте.

(ii) Вставка обладает существенной степенью внутренней законченности. При максимальной выраженности этого признака во вставке реализуется самостоятельная иллокутивная функция, отдельная от иллокутивной функции объемлющего контекста, - см. пример (1), в котором во вставку помещен оценочный комментарий говорящего, внешний по отношению к миру рассказа. Типичным интонационным коррелятом иллокутивной независимости выступает нисходящий или ровный акцент в главном акценте. Если же вставка не обладает полноценной иллокутивной силой, наличие нисходящего или ровного акцента становится главным фактором, противопоставляющим вставку окружающему контексту. Нередко при этом падение частоты основного тона не достигает нижнего для данного говорящего уровня; в транскриптах (2) и (3) на это указывают запятые (а не точки) перед закрывающимися скобками.

Заметной интонационной характеристикой прототипической вставки также может выступать последовательное понижение частоты основного тона на протяжении всего вставленного фрагмента - см. тонограмму примера (4) на рис. 2. Вставка, состоящая из двух клауз (Е015-Е016), произносится в «сплющенном» тональном диапазоне и с практически равномерным, пологим падением. Такое оформление резко контрастирует с интонационными фигурами, реализованными в левом и правом контексте и включающими чередования восходящих и нисходящих тональных движений.

\footnotetext{
6 Другой подход представлен, в частности, в [Богданова 2011], где прямо утверждается, что интонационные критерии не позволяют выделить вставки в контексте и что вставки не чувствительны к границам устных «предложений».
} 
(4) Pic-RUS_01-f_Ski-T

\begin{tabular}{|c|c|c|}
\hline 30.15 & E014 & 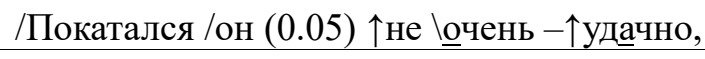 \\
\hline 32.14 & $\mathrm{p}-013$ & $(0.56)$ \\
\hline 32.70 & E015 & (так как был \пьянный, \\
\hline 33.71 & $\mathrm{p}-014$ & $(0.15)$ \\
\hline 33.85 & E016 & а за рулём как известно ^нельз多 пить, ) \\
\hline 35.55 & $\mathrm{p}-015$ & $(0.86)$ \\
\hline 36.41 & E017 & /и /попал в $\downarrow-$ реанимацию! \\
\hline
\end{tabular}

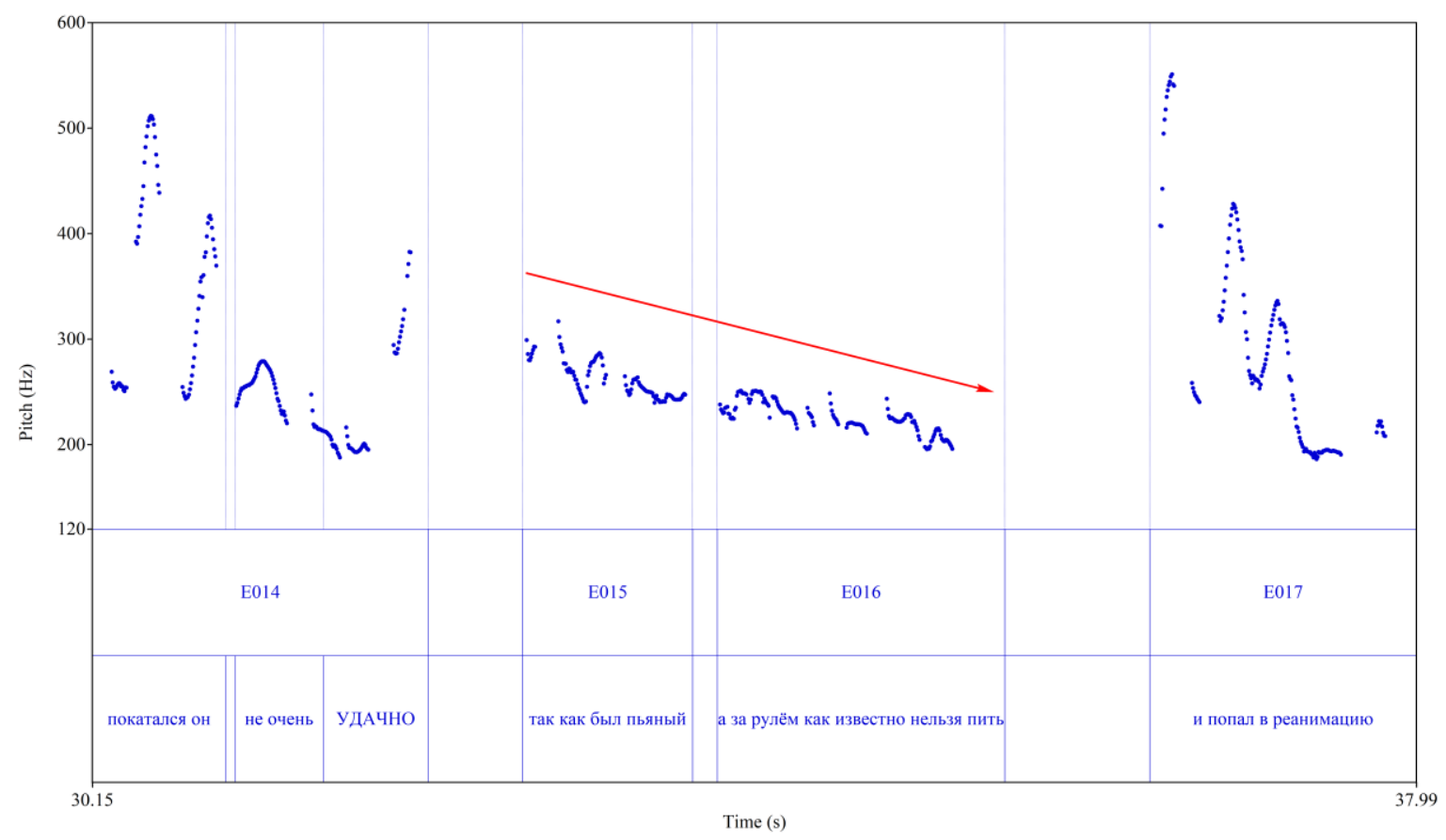

Рис. 2. Тонограмма примера (4). Стрелкой выделено последовательное понижение частоты основного тона на протяжении двух вставленных ЭДЕ

Далеко не все вставки характеризуется столь же явной интонационной обособленностью, как в примере (4). Тем не менее в целом немногим менее половины всех обнаруженных в корпусе случаев удовлетворяют двум приведенным выше условиям (см. далее табл. 1). Оставшиеся примеры тем или иным образом отклоняются от указанного прототипа.

\section{2 «Свободные» вставки}

В отличие от вставок в примерах (1)-(3), «свободные» вставки либо содержательно соотносятся со всем объемлющим контекстом целиком (а не с его отдельными элементами), либо вовсе чужеродны контексту. В [Dehé 2014: 8ff] для таких случаев используются ярлыки floating и detached parentheticals. Пример вставки, полностью выпадающей из основной линии изложения, приведен в (5). В строке N-vE233 говорящая начинает отвечать на вопрос собеседницы о внешности персонажа обсуждаемого фильма, проецируя продолжение посредством стандартной восходящей интонации. В это же время альтернативную попытку ответить на тот же вопрос предпринимает третий участник записи. Не желая уступать право хода, говорящая приостанавливает ответ на вопрос, предлагает третьему участнику повременить с его версией (строки N-vE234-235), а уже затем, в строке N-vE236, приступает к ранее проецированному продолжению7 .

\footnotetext{
${ }^{7}$ В [Богданова 2011] аналогичные примеры определяются как металингвистические вставные конструкции и рассматриваются в ряду характерных для спонтанной речи типов. Как видно из табл. 1, в наших данных такие примеры относительно редки, что, разумеется, не отменяет их содержательной специфики.
} 
(5) Pears $22 \mathrm{~N}$

\begin{tabular}{|c|c|c|c|}
\hline 502.38 & N-vE233 & Он такой очень /смешной, & $\begin{array}{l}\text { Обращается к Пере- } \\
\text { сказчице }\end{array}$ \\
\hline 503.65 & pN-054 & $(0.25)$ & \\
\hline 503.90 & $\mathrm{~N}-\mathrm{vE} 234$ & (/Можно я расскажу ${ }^{\mathrm{h}}$ ? & $\begin{array}{l}\text { Обращается к Ком- } \\
\text { ментатору }\end{array}$ \\
\hline 504.93 & $\mathrm{~N}-\mathrm{vE} 235$ & А потом ты ко= || /откомментируешь.) & \\
\hline 506.57 & $N-v L 007$ & $\{$ laugh 0.64$\}$ & \\
\hline 507.21 & $\mathrm{~N}-\mathrm{vE} 236$ & у него такая объёмная /шевелюра, & $\begin{array}{l}\text { Обращается к Пере- } \\
\text { сказчице }\end{array}$ \\
\hline 508.93 & N-vN052 & (ч 0.33$)$ & \\
\hline 509.25 & $\mathrm{~N}-\mathrm{vE} 237$ & (Вㅡㅜ,) & \\
\hline 509.66 & $\mathrm{~N}-\mathrm{vE} 238$ & и /усы $==$ & \\
\hline
\end{tabular}

\section{3 Парентетическое вот}

В строке N-vE237 примера (5) представлен еще один тип вставки - парентетическое вот. Полноударная реализация дискурсивного маркера вот стандартно используется для указания на «завершение структурно значимого фрагмента текста и переход к следующему» [Дараган 2003]. Если вот следует за финальной ЭДЕ, считать его вставкой - согласно приведенному в разделе 3 определению - нет оснований. Если же подытоживаемый дискурсивный фрагмент произносится с интонацией незавершенности, то вот может иметь парентетическую природу. Именно так происходит в примере (5). Урегулировав коллизию с правом очередности, говорящая возвращается к описанию персонажа и произносит ЭДЕ N-vE236 - вторую после N-vE234 в серии нефинальных единиц основной линии. Прежде чем продолжить, она реализует вставочное вот, давая таким образом понять, что не планирует более говорить о прическе, а намеревается перейти к другим аспектам внешности обсуждаемого персонажа - что и делает в строке N-vE238. (Дальнейшее развитие прерывается по причинам, не имеющим отношения к рассматриваемым в работе явлениям.)

Парентетическое вот практически всегда произносится с нисходящим или ровным акцентом. При этом оно не соотносится с каким-либо отдельным элементом левого или правого контекста, а указывает на то, как говорящий в целом оценивает формируемую локальную структуру. По предварительным наблюдениям, частота использования подытоживающего вот (как в финальной, так и в парентетической позиции) обусловлена индивидуальной манерой речи: одни говорящие прибегают к этому средству структурирования речевого потока регулярно, другие - редко или практически никогда. Так, из 51 вхождения парентетического вот в трех записях корпуса «Рассказы и разговоры о грушах» 21 случай обнаруживается в речи одной участницы. Отмечу также, что парентетическую функцию способны выполнять и некоторые другие дискурсивные маркеры или их сочетания: $н y$, ну вот, значит и др. Однако в рассмотренном материале вот частотность таких случаев значительно уступает частотности парентетического вот.

\section{4 Псевдопарентетические вставки}

Наиболее заметное отклонение от рассмотренного в разделе 4.1 прототипа наблюдается при произнесении вставленных единиц с восходящим движением тона. В этом случае возникает противоречие между содержательной ролью вставки в локальной структуре и ее интонационным оформлением. Так, в примере (6) основная линия изложения связывает ЭДЕ N-vE173 и N-vE175 - два нерестриктивных придаточных определительных, сочиненных между собой и имеющих общее подлежащее. В первом из них при помощи нисходяще-восходящего акцента типа ИК-4 проецируется продолжение, реализуемое во втором. Располагающаяся между ними ЭДЕ N-vE174 демонстрирует стандартные структурные и содержательные характеристики вставки: в ней не содержится намеченного ранее продолжения основной линии, она уточняет элемент левого контекста и составляет побочный дискурсивный шаг. Однако интонационно эта строка 
оформлена так же, как левый контекст - в ней тоже реализуется нисходяще-восходящее тональное движение, как если бы она была полноценным элементом основной линии. Как можно заметить, в транскриптах такие «псевдопарентетические» единицы не заключаются в скобки.

(6) Pears04N

\begin{tabular}{|c|c|c|}
\hline 287.59 & $\mathrm{~N}-\mathrm{vE} 172$ & 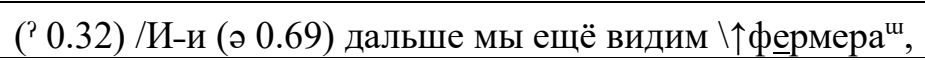 \\
\hline 290.92 & $\mathrm{pN}-066$ & $(0.69)$ \\
\hline 291.61 & N-vE173 & 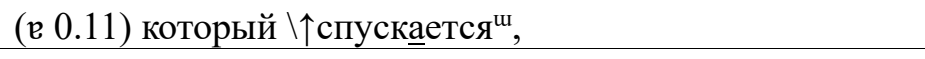 \\
\hline 293.21 & $\mathrm{pN}-067$ & $(0.38)$ \\
\hline 293.59 & N-vE174 & c \^лестницы, \\
\hline 294.23 & $\mathrm{~N}-\mathrm{vE} 175$ & и видит что-о /'одной из корзин не $\backslash$ хватае $\mathrm{T}^{\mathrm{h}}$. \\
\hline
\end{tabular}

Количественное распределение рассмотренных в разделе 4 типов представлено в табл. 1. Как видно, к ядерному типу относится немногим менее половины случаев, следующими по частотности оказались конструкции с парентетическим вот, далее - конструкции с псевдопарентетическими вставками. Также можно отметить, что три наиболее часто встречающихся типа по-разному распределены по значениям параметра «уровень дискурсивной структуры»: парентетические вот встречаются только между ЭДЕ; для ядерного типа расположение вставки внутри ЭДЕ возможно, но значительно менее частотно, чем позиция между ЭДЕ; псевдопарентетические вставки с равной частотой возникают между и внутри ЭДЕ.

\begin{tabular}{|l|c|c|c|}
\hline \multirow{2}{*}{ Тип вставочной конструкции } & \multicolumn{3}{|c|}{ Уровень дискурсивной структуры } \\
\cline { 2 - 4 } & Между ЭДЕ & Внутри ЭДЕ & Всего \\
\hline Ядерный тип & 100 & 14 & $114(48.3 \%)$ \\
\hline Со «свободными» вставками & 8 & 2 & $10(4.2 \%)$ \\
\hline С парентетическим вот & 68 & 0 & $68(28.8 \%)$ \\
\hline $\begin{array}{l}\text { С псевдопарентетическими } \\
\text { вставками }\end{array}$ & 19 & 20 & $39(16.5 \%)$ \\
\hline Прочие & & 3 & $5(2.1 \%)$ \\
\hline ВСЕГО & 2 & 39 & $236(100 \%)$ \\
\hline
\end{tabular}

Табл. 1. Количественное распределение типов конструкций с дискурсивными вставками в проанализированном корпусе: общее и отдельные по уровням дискурсивной структуры

\section{5 Темп и регистр во вставках: предварительный анализ}

В литературе, посвященной парентезе, неоднократно отмечалось, что вставки характеризуются рядом просодических признаков: тихим и ускоренным произнесением, сниженным или сжатым тональным регистром и др. [Crystal 1969; Цеплитис 1974; Kutik et al. 1983; Bolinger 1989; Wichmann 2001; Гавриленко 2004; Кибрик, Подлесская 2010; Dehé 2014; и др.]. Для проверки этих утверждений на корпусном материале в рамках настоящего исследования был проведен дополнительный акустический анализ двух просодических характеристик вставок относительно левого и правого контекста: темпа и тонального регистра.

В качестве меры темпа была выбрана средняя продолжительность слога в миллисекундах, в качестве меры тонального регистра - ширина тонального диапазона, которая рассчитывалась как разность между 90-м и 10-м процентилем в ряду значений ЧОТ, определяемых в объекте Pitch программы Praat. Соответственно, под коэффициентом ускорения понималось отношение темпа во вставке к темпу в левом / правом контексте; под коэффициентом сжатия тонального диапазона - отношение ширины диапазона во вставке к ширине диапазона в левом / правом контексте. Так, в приведенном выше примере (4) коэффициент ускорения вставки так как был пьяный, а за рулём как известно нельзя пить по отношению как к левому, так и к правому контексту составил 1.10; сжатие тонального диапазона составило 3.13 по отношению к левому контексту и 3.37 по отношению к правому (ср. наблюдаемый на тонограмме контраст на рис. 2; указанная разница соответствует 8-9 полутонам). 

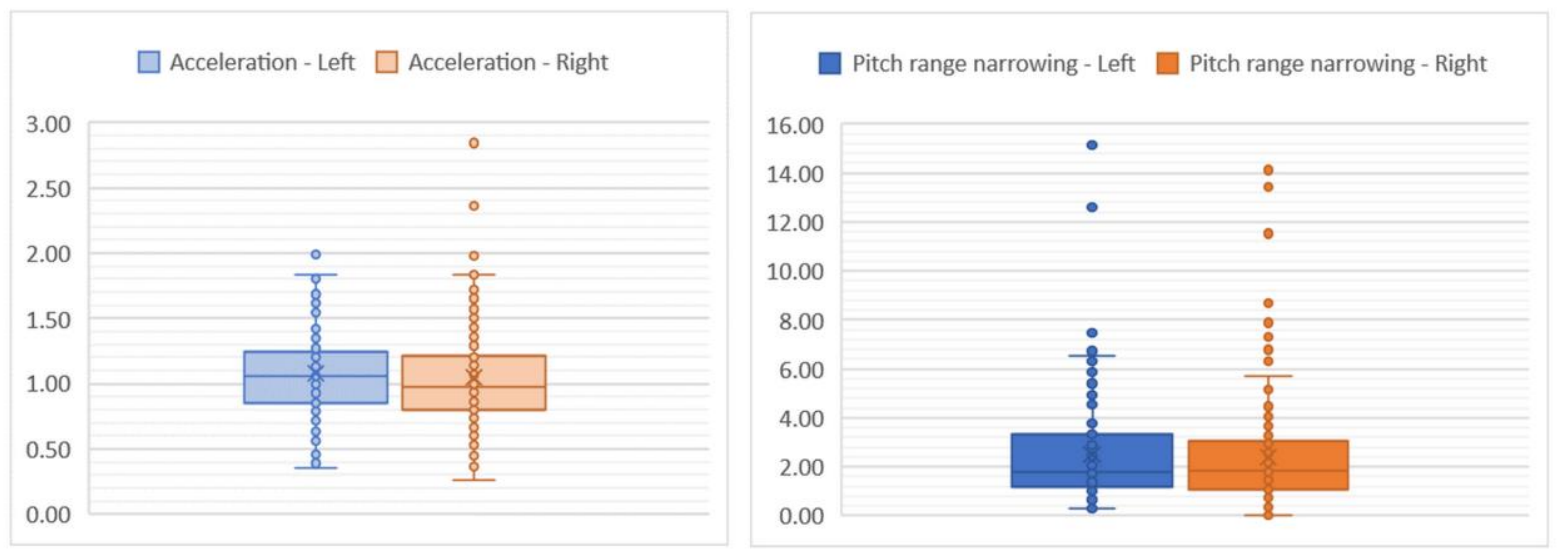

Рис. 3. Диаграммы размаха просодических характеристик вставок. Слева направо: коэффициент ускорения относительно левого контекста, коэффициент ускорения относительно правого контекста, коэффициент сжатия тонального диапазона относительно левого контекста, коэффициент сжатия тонального диапазона относительно правого контекста

Поскольку в конструкциях с парентетическим вот вставки состоят всего из одного слога, эти примеры были исключены из выборки. Для оставшихся 168 примеров были подсчитаны значения четырех коэффициентов; диаграммы размаха представлены на рис. 3. При проверке одновыборочным t-тестом статистическую значимость показали сжатие тонального диапазона относительно левого и правого контекста ( $\mathrm{M}=2.50$ и 2.39 соответственно; $p<0.0001$; умеренный размер эффекта по $\mathrm{d}$ Коэна), а также ускорение относительно левого контекста (M $=1.08 ; p=0.002$; малый размер эффекта). Значимых корреляций между темповыми и тональными параметрами не обнаружено, что можно интерпретировать как указание на независимость этих двух просодических характеристик.

Кроме того, были выявлены некоторые предварительные закономерности, связанные с параметрами классификации вставочных конструкций, рассмотренными выше в разделах 3 и 4:

- Сжатие тонального регистра относительно правого контекста статистически значимо для вставок ядерного типа, но не для псевдопарентетических вставок.

- Вставки внутри ЭДЕ (см. пример (3)) демонстрируют бо́льшую тенденцию к ускорению относительно левого контекста, чем вставки между ЭДЕ. При этом данное различие статистически значимо только для псевдопарентетических вставок $(p=0.02)$, но не для вставок ядерного типа $(p=0.27)$.

- Для сжатия тонального диапазона наблюдается противоположная картина: эта характеристика более заметно реализуется во вставках между ЭДЕ, чем во вставках внутри ЭДЕ $(p=0.02)$.

Содержательная интерпретация полученных количественных результатов, по всей видимости, станет возможна при увеличении объема выборки. Предварительно можно заключить, что из двух рассмотренных просодических характеристик вставок на нашем материале более последовательно проявляется сжатие тонального диапазона. Несколько более подробный анализ просодических характеристик вставок ядерного типа содержится в [Коротаев 2021].

\section{6 Заключение}

В настоящей работе вставка, или парентеза, рассматривается как явление дискурсивного уровня. Вставочная природа языковой единицы - это характеристика конкретной дискурсивной ситуации, в которой вставка противопоставляется окружающему контексту. Соответственно, интегральные и дифференцирующие свойства парентетических конструкций обусловлены (а) внутренними свойствами объемлющего контекста; (б) внутренними свойствами вставленных единиц; (в) характером взаимоотношения вставки и контекста. При определении понятия на материале неподготовленной устной речи за основу было взято представление о вставке как об отдельном 
дискурсивном шаге, в котором временно откладывается проецированное в левом контексте продолжение основной линии изложения. Таким образом, парентеза становится компонентом одной из возможных стратегий «разрешения» дискурсивной незавершенности, см. [Коротаев 2018].

Согласно собранным корпусным данным, прототипические вставки содержательно соотносятся с тем или иным элементом левого контекста, а также произносятся с нисходящим или ровным тональным акцентом. Отклонения от этого прототипа возможны по обоим параметрам, но наиболее заметным оказывается использование во вставке восходящей интонации - если она не связана с выражением самостоятельного иллокутивного значения. Просодические характеристики вставок, противопоставляющие их левому и правому контексту, включают в себя ускоренное произнесение и сжатие тонального диапазона, при этом второе свойство реализуется в наших данных более рельефно, чем первое.

\section{Благодарности}

Исследование выполнено при финансовой поддержке РНФ, проект № 17-18-01184.

\section{Литература}

[1] Auer, Peter. (2005). Projection in Interaction and Projection in Grammar, Text - Interdisciplinary Journal for the Study of Discourse, Vol. 25(1), pp. 7-36. https://doi.org/10.1515/text.2005.25.1.7.

[2] Boersma, Paul, Weenink, David (2018). Praat: Doing phonetics by computer (6.0.43) [Computer software]. Access mode: https://www.fon.hum.uva.nl/praat/.

[3] Bogdanova, Natalia V. (2011). Parenthetical constructions in spoken spontaneous monologues [Vstavnye konstrukcii v zvučaščem spontannom monologe], Studies in Speech Culture [Voprosy kul'tury reči], Iss. 10, pp. 204-212.

[4] Bolinger, Dwight (1989). Intonation and Its Uses: Melody in Grammar and Discourse. Stanford, Stanford University Press.

[5] Ceplītis, Laimodts (1974). Analysis of speech intonation [Analiz rečevoj intonacii]. Riga, Zinātne.

[6] Crystal, David (1969). Prosodic Systems and Intonation in English. Cambridge, Cambridge University Press.

[7] Daragan, Yulia V. (2003). Parasitism or symbiosis? How speakers deal with communication failures and verbal means they use to do that [Parazitizm ili simbioz: Mexanizm preodolenija kommunikativnyx sboev $\mathrm{i}$ obsluživajuščie ego verbal'nye sredstva ], Computational Linguistics and Intellectual Technologies: Proceedings of the International Conference "Dialogue" [Komp'juternaja lingvistika i intellektual'nye texnologii: po materialam ežegodnoj meždunarodnoj konferencii "Dialog”], pp. 166-178.

[8] Dehé, Nicole (2014). Parentheticals in Spoken English: The Syntax-Prosody Relation. Cambridge, Cambridge University Press. https://doi.org/10.1017/CBO9781139032391

[9] Dehé, Nicole, Kavalova, Yordnaka (Eds.) (2007). Parentheticals. John Benjamins. https://doi.org/10.1075/la.106

[10] Gavrilenko, Irina I. (2004). Supplementary information in academic texts: Semantic, syntactic, and prosodic features (based on Russian data) [Dopolnitel'naja informacija v naučnyx tekstax: Semantičeskie, sintaksičeskie i prosodičeskie osobennosti (na materiale russkogo jazyka)], PhD Dissertation Abstract. Moscow, Moscow State University.

[11] Kavalova, Yordanka (2007). And-parenthetical clauses, N. Dehé, Y. Kavalova (eds.) Parentheticals. John Benjamins, pp. 145-172. https://doi.org/10.1075/la.106.09kav

[12] Kibrik, Andrej A. (2018). Russian multichannel discourse. Part II. Corpus development and avenues of research [Russkij mul'tikanal'nyj diskurs. Čast' II. Razrabotka korpusa i napravlenija issledovanij], Psychological Journal [Psixologičeskij Žurnal], Vol 39(2), pp. 79-90. https://doi.org/10.7868/80205959218020083

[13] Kibrik, Andrej A., Korotaev, Nikolay A., Podlesskaya, Vera I. (2020). Russian spoken discourse: Local structure and prosody, S. Izre'el, H. Mello, A. Panunzi, T. Raso (eds.) In search of basic units of spoken language: A corpus-driven approach. John Benjamins, pp. 37-76. https://doi.org/10.1075/sc1.94.01kib

[14] Kibrik, Andrej A., Podlesskaya, Vera I. (2010). Parenthetical construction in spoken discourse [Vstavočnye konstrukcii v ustnom diskurse], V. Z. Demyankov, V. Ja. Porxomovskij (eds.) In language and culture: Sound, sign, meaning. For the 70th birthday of Viktor A. Vinogradov [V prostranstve jazyka i kul'tury: Zvuk, znak, smysl. Sbornik statej v čest' 70-letija V. A. Vinogradova]. Moscow, LSC, pp. 87-99.

[15] Kobozeva, Irina M. (1999). On two types of introductory constructions with parenthetical verbs [O dvux tipax vvodnyx konstrukcij s parentetičeskim glagolom], E. V. Raxilina, Ja. G. Testelets (eds.) Typology and Lingustic theory: From description to explanation. For the 60th birthday of Aleksandr E. Kibrik [Tipologija i teorija jazyka: Ot opisanija k ob"jasneniju. K 60-letiju A. E. Kibrika]. Moscow, LSC, pp. 539-543. 
[16] Kodzasov, Sandro V. (2009). Studies in Russian Prosody [Issledovanija v oblasti russkoj prosodii]. Moscow, LSC.

[17] Korotaev, Nikolay A. (2018). How intonation structure spoken narratives: Non-final phase contexts [Intonacionnaja struktura ustnogo rasskaza v kontekste nezaveršennosti], Computational Linguistics and Intellectual Technologies: Proceedings of the International Conference "Dialogue" [Komp'juternaja lingvistika i intellektual'nye texnologii: po materialam ežegodnoj meždunarodnoj konferencii “Dialog”], Vol. 17(24), pp. 342-356.

[18] Korotaev, Nikolay A. (2021). Tempo and pitch properties of parenthetical constructions in spontaneous Russian discourse [Temp i tonal'nyj registr v konstrukcijax so vstavkami v nepodgotovlennom ustnom diskurse], Analysis of the Russian colloquial speech [Analiz razgovornoj russkoj reči], St. Petersburg. In print.

[19] Kutik, Elanah J., Cooper, William E., Boyce, Suzanne (1983). Declination of fundamental frequency in speakers' production of parenthetical and main clauses, The Journal of the Acoustical Society of America, Vol. 73(5), pp. 1731-1738. https://doi.org/10.1121/1.389397

[20] Mazeland, Harrie (2007). Parenthetical sequences, Journal of Pragmatics, Vol. 39(10), pp. 1816-1869. https://doi.org/10.1016/j.pragma.2007.05.005

[21] McCawley, James D. (1982). Parentheticals and discontinuous constituent structure, Linguistic Inquiry, Vol. 13(1), pp. 91-106.

[22] Paducheva, Elena V. (2010). Semantic studies: Semantics of tense and aspect in Russian; Semantics of the narrative [Semantičeskie issledovanija: Semantika vremeni i vida v russkom jazyke; Semantika narrativa] (2nd ed.). Moscow, LSC.

[23] Schegloff, Emanuel A. (2007). Sequence Organization in Interaction: A Primer in Conversation Analysis, Vol. 1. Cambridge, Cambridge University Press. https://doi.org/10.1017/CBO9780511791208

[24] Urmson, James O. (1952). Parenthetical verbs, Mind, Vol. 61(244), pp. 480-496.

[25] Wichmann, Anne (2001). Spoken parentheticals, K. Aijmer (Ed.), A Wealth of English: Studies in Honour of Goran Kjellmer. Gothenburg, Gothenburg University Press, pp. 177-193.

[26] Yanko, Tatiana E. (2001). Communicative strategies of Russian speech [Kommunikativnye strategii russkoj reči]. Moscow, LSC.

[27] Yanko, Tatiana E. (2008). Intonational strategies in spoken Russian from a comparative perspective [Intonacionnye strategii russkoj rechi v tipologicheskom aspekte]. Moscow, LSC. 